\title{
Mapeamento sistemático sobre a avaliação da aprendizagem no ensino de lógica de programação no Ensino Médio
}

\author{
Alex Lacerda Gomes Loiola ${ }^{1}$, Francisco Kelsen de Oliveira ${ }^{2}$ \\ Mestrado Profissional Em Educação Profissional Tecnológica (IFSertãoPE) ${ }^{1,2}$; Salgueiro - PE - Brasil \\ https://orcid.org/0000-0002-5068-344X'1 https://orcid.org/0000-0002-7382-3206²; \\ alexlgloiola@gmail.com ${ }^{1}$; francisco.oliveira@ifsertao-pe.edu.br ${ }^{2}$
}

Resumo. Este estudo tem o propósito de fazer um mapeamento sistemático sobre as estratégias ou metodologias de avaliação de aprendizagem utilizadas no ensino de lógica de programação para estudantes do Ensino Médio. Para realização do nosso intento, utilizamos a metodologia de Mapeamento Sistemático de Literatura, que possibilita a busca metódica e ordenada de estudos primários e garante a sua reprodutibilidade ou verificabilidade dos resultados por outros pesquisadores. Os estudos que tivemos acesso não trouxeram resultados sobre as estratégias ou metodologias de avaliação da aprendizagem mais eficientes para a avaliação do ensino de programação. Esse fato nos mostra que ainda não há um questionamento científico sobre a eficiência dos métodos ou estratégias de avaliação da aprendizagem no ensino de programação para estudantes do Ensino Médio.

Palavras-chave: avaliação da aprendizagem; lógica de programação; Ensino Médio

\section{Systematic mapping on learning assessment in the teaching of programming logic in high school}

\begin{abstract}
This study has the purpose of making a systematic mapping on the strategies or methodologies of learning evaluation used in the teaching of programming logic for high school students. In order to achieve our intent, we use the Systematic Literature Mapping methodology, which enables the methodical and orderly search for primary studies and guarantees their reproducibility or verifiability of the results by other researchers. The studies that we had access to did not bring results on the most efficient learning assessment strategies or methodologies for the assessment of programming teaching. This fact shows us that there is still no scientific question about the efficiency of methods or strategies for assessing learning in teaching programming for High School students.
\end{abstract}

keywords: assessment; logic programming; High School

\section{Introdução}

A avaliação da aprendizagem escolar é tema que vem sendo discutido por pesquisadores da educação, tais como Luckesi (2011), Romão (2011) e Perrenoud (1999). A avaliação é um dos componentes do ato pedagógico e, como tal, precisa ser objeto de reflexão e planejamento adequado por parte dos professores, bem como fazer parte das estratégias institucionais para garantir a qualidade do ensino e promover $\mathrm{o}$ desenvolvimento dos educandos.

É fundamental que a avaliação da aprendizagem não aconteça como uma atividade burocrática na rotina da escola e do professor, ou seja, simplesmente como aplicação de um dado número de provas semestrais, como estabelecido pelo projeto pedagógico da escola, para gerar notas e classificar os estudantes como aprovados ou reprovados no final do ano letivo. Esse é um tipo de prática avaliativa que se confunde com verificação e pouco contribui para a construção das aprendizagens.

De acordo com Luckesi (2011, p. 13), longe de se confundir com um ato burocrático de verificação, a avaliação da aprendizagem é uma atividade de investigação V. $19 \mathrm{~N}^{\circ} 1$, julho, 2021 RENOTE 
qualitativa sobre um objeto, que no caso são as aprendizagens. Nesse sentido, a avaliação não se constitui como um fim em si mesma, mas como uma estratégia metódica e reflexiva para tomar decisões pedagógicas e administrativas para alcançar com sucesso os objetivos traçados no projeto educativo.

Se a avaliação da aprendizagem é uma atividade de investigação constante sobre a qualidade das aprendizagens, a questão que se coloca é justamente quais são os instrumentos mais adequados para proceder com a investigação e realizar um bom diagnóstico das aprendizagens em sala de aula, possível de ser utilizado para o planejamento e a tomada de decisão sobre as estratégias de ensino, no sentido garantir o sucesso escolar com a aprendizagem dos estudantes.

Isso nos motivou à realização dessa pesquisa, buscando mapear sistematicamente as discussões sobre estratégias e metodologias de avaliação da aprendizagem aplicadas no ensino de lógica de programação para a educação básica, particularmente os cursos de Ensino Médio técnico que tenham essa disciplina no currículo.

\section{A avaliação de aprendizagem na área de ensino de computação}

A área de computação é bastante instigante para o público em geral e para aqueles que resolvem se aprofundar na área via estudo formal. No entanto, o ensino dos conteúdos de programação e algoritmo, conteúdos fundamentais para o desenvolvimento do estudante na área de computação, é permeado de desafios. Acreditamos que esses desafios se tornam ainda mais presentes nas propostas de ensino de computação para jovens de 14 a 17 anos que estão no Ensino Médio, uma vez que, esses educandos estão estudando, ao mesmo tempo, os conhecimentos que são base para o pensamento formal, considerado pré-requisito para o processo de formalização de problemas em nível computacional.

Para Brito e Madeira (2015), grande parte dos estudantes apresentam dificuldades na formalização de problemas que se apresentam na realidade para a linguagem computacional. Os autores atribuem essas dificuldades tanto à complexidade dos conteúdos da disciplina de programação e do esforço com o tempo de práticas que é necessária para o desenvolvimento dos estudantes, quanto à deficiência dos estudantes em conhecimentos básicos em matemática e à pouca capacidade de resolução de problemas. De acordo com Raabe e Silva (2005), o alto nível de abstração exigido é um dos fatores de desmotivação e desistência dos estudantes. Além do que, como asseveram Jesus e Brito (2009 p. 132) "[...] essas habilidades e competências são pré-requisitos à maioria das disciplinas trabalhadas em cursos de Computação e Informática."

O objetivo do trabalho com os conteúdos de algoritmo e lógica de programação é, basicamente, desenvolver nos estudantes as ferramentas conceituais para que sejam capazes de programar. A tarefa de programar é a capacidade de, mediante um problema, ser capaz de desenvolver uma resposta (programa) em nível computacional, utilizando um conjunto de passos ou instruções lógicas (algoritmo), na linguagem apropriada para a comunicação com as máquinas (linguagens de programação), de modo a fazer com que o computador realize uma determinada função.

No entanto, desenvolver as competências que permitem que o sujeito seja capaz de oferecer respostas computacionais para os problemas postos no mundo real não é uma tarefa fácil. O processo para a formação de novos programadores encontra dificuldades justamente por constatar que parte considerável dos estudantes matriculados em disciplinas introdutórias apresentam considerável dificuldade de compreensão e aplicação dos conceitos mais abstratos de programação ( PIMENTEL, FRANÇA, et al., 2003).

Para compreender a especificidade das disciplinas de algoritmo e programação, Jesus e Brito (2009) nos dizem que é necessário ter em consideração os conhecimentos que estão intrinsecamente relacionados a essas disciplinas: lógica, algoritmo, linguagem 
de programação. De acordo com Forbellone e Eberspächer (2000, p.01): "Podemos relacionar a lógica com a 'correção do pensamento', pois uma de suas preocupações é determinar quais operações são válidas e quais não são, fazendo análises das formas e leis do pensamento". Ainda segundo Forbellone e Eberspächer (2000, p.02), a lógica de programação seria o uso correto e ordenado da razão e do raciocínio, de acordo com uma simbolização formal na programação, com o objetivo de alcançar "[...] a racionalidade e o desenvolvimento de técnicas que cooperem para a produção de soluções logicamente válidas e coerentes, que resolvam com qualidade os problemas os problemas que se deseja programar."

O conceito de algoritmo é definido por Jesus e Brito (2009, p.133), como uma sequência de passos, logicamente encadeados, com a finalidade de atingir um objetivo delimitado. Por sua vez, a linguagem de programação é a ferramenta que possibilita a implementação do software.

A atividade de programação requer do estudante o desenvolvimento do raciocínio lógico como uma das condições para a construção de algoritmos. Além do domínio lógico para a formalização dos algoritmos, o estudante precisa compreender o funcionamento da sintaxe e semântica de uma linguagem de programação, para que possa efetivamente implementar uma solução algorítmica possível de ser lida pelo computador (JESUS e BRITO, 2009).

A capacidade de trabalhar no nível do pensamento abstrato e lógico é uma exigência para que o aluno possa desenvolver de forma satisfatória os conhecimentos e competências na área de algoritmo e lógica de programação. Por outro lado, a atividade de ensino de algoritmo e lógica de programação exige, do docente, a capacidade de utilizar metodologias que favoreçam o desenvolvimento das competências necessárias para o êxito na disciplina, bem como ser capaz de despertar nos estudantes o interesse pelo conteúdo ( RIBEIRO, BITTENCOURT e SANTANA, 2019).

As dificuldades dos estudantes são, em grande parte, causadas pelo nível de abstração que o conteúdo exige, além da dificuldade em mobilizar conhecimentos de outras áreas para compreender os problemas propostos nas disciplinas de computação, tal como apontado por Raabe e Silva (2005).

Os pesquisadores que trabalham com ensino de computação, apontam também para a dificuldade que os docentes possuem de trabalhar os conhecimentos dessas disciplinas no nível de compreensão dos estudantes e utilizando metodologias de ensino e meios tecnológicos adequados para os objetivos propostos e que despertem a curiosidade dos estudantes pelo conteúdo trabalhado (JESUS e BRITO, 2009).

O uso de exemplos hipotéticos pode ser um recurso válido em alguns momentos, desde que os estudantes já possuam capacidade de domínio dos conteúdos que permita acompanhar pelo raciocínio a formulação da hipótese e saber quais soluções estão envolvidas na explicação. Dessa forma, a hipótese se torna uma ferramenta a mais de exploração das possibilidades de aplicação e desenvolvimento do conhecimento. Para fazer uso adequado desse tipo de estratégia, o professor precisa conhecer e desenvolver um conjunto de ferramentas e estratégias que possibilitem ao aluno se desenvolver na aprendizagem dos conhecimentos da área. Para isso, precisa ir além da apresentação de exemplos, hipóteses ou soluções que ele encontrou para o próprio aprendizado de programação.

Por isso, é necessário se apropriar das teorias que tratam dos processos de ensino e aprendizagem humana, bem como das ferramentas pedagógicas adequadas e próprias da sua área de ensino para que tenha, tanto domínio conceitual da matéria, quanto conhecimento didático-pedagógico para promover o desenvolvimento dos estudantes ( JESUS e BRITO, 2009). 
No mesmo sentido, a apresentação de paradigmas diferentes de computação aos estudantes, sem levar em consideração a sua capacidade de abstração e o seu nível de desenvolvimento intelectual, torna o processo de aprendizagem muito cansativo e desestimulante (RIBEIRO, BITTENCOURT e SANTANA, 2019).

Os trabalhos na área de ensino de programação têm abordado, como aponta Delgado et. al. (2005), as competências relacionadas à aprendizagem dos conteúdos de algoritmo e programação e a pesquisa de metodologias para o ensino dos conteúdos dessas disciplinas. No entanto, as discussões sobre a avaliação da aprendizagem em programação e nos conteúdos de algoritmo e lógica de programação não estão presentes na mesma intensidade. Isso pode sinalizar que não está tão evidente para os pesquisadores da área que a avaliação da aprendizagem é também um componente do ato pedagógico no ensino, ou seja, que a avaliação da aprendizagem é uma ferramenta que deve ser utilizada pelo professor juntamente com as metodologias de ensino, como estratégia para o desenvolvimento dos estudantes.

A tarefa de identificar o nível de aprendizagem dos estudantes é algo bastante complexo para o professor. Mas, não sendo capaz de detectar as dificuldades de aprendizagem no tempo correto, no ensino de conteúdos mais básicos, esses problemas de aprendizagem acabam por dificultar o desenvolvimento do estudante em conteúdos e atividades mais complexas que envolvem a habilidade de programação ( ROCHA, FERREIRA, et al., 2010).

Isso aponta para a necessidade de aprofundar a pesquisa sobre a avaliação da aprendizagem dos conteúdos e competências das disciplinas de algoritmo e lógica de programação, que são fundamentais para o desenvolvimento das habilidades de programação de computadores. Somente com o desenvolvimento de eficientes estratégias de avaliação da aprendizagem é possível acompanhar e fomentar o desenvolvimento dos estudantes na área.

\section{Material e Métodos}

$\mathrm{O}$ presente trabalho de mapeamento segue um modelo de pesquisa científica denominado Revisão Sistemática de Literatura (RSL), que consiste na busca e análise sistemática de estudos primários sobre um determinado tema, seja para encontrar a resposta ou solução mais adequada para determinado problema na literatura científica (RSL), ou, no caso do mapeamento sistemático, verificar o que já foi publicado de literatura científica sobre um assunto específico.

O mapeamento sistemático de literatura deve seguir a mesma rigorosidade nas buscas dos estudos primários que uma RSL. Como aponta Okoli (2019), esse tipo de pesquisa deve seguir e explicitar os procedimentos metodológicos utilizados pelo pesquisador para chegar aos resultados encontrados, de forma a tornar o trabalho reprodutível e verificável por outros pesquisadores interessados no tema.

Como todo empreendimento de pesquisa científica, o mapeamento sistemático tem como ponto de partida a formulação de uma pergunta de pesquisa adequadamente construída para essa finalidade. Para garantir o caráter sistemático, explícito e reprodutível do trabalho de revisão, os pesquisadores devem deixar claros os passos e procedimentos utilizados para a realização da coleta de informações nos estudos primários, por meio da elaboração de um protocolo de pesquisa. É justamente isso que buscaremos fazer neste tópico ao explicitar a pergunta de pesquisa, as bases de dados, os critérios de elegibilidade, as definições de estratégias de busca, o processo de triagem e seleção dos estudos, bem como o processo de extração dos dados.

A pergunta que guiou a nossa investigação foi a seguinte: "Quais as estratégias ou metodologias de avaliação de aprendizagem escolar utilizadas no ensino de lógica de 
programação para estudantes do Ensino Médio?" Com essa pergunta, tínhamos o intuito de mapear, na literatura científica, os modelos ou estratégias de avaliação da aprendizagem escolar utilizados no ensino de lógica de programação para os estudantes da educação básica, que fundamentalmente ocorre em cursos técnicos de nível médio, como o de técnico em informática integrado ao Ensino Médio da rede federal de Educação Profissional e que tem a referida disciplina na grade curricular.

Ao definir a pergunta de pesquisa do trabalho, passamos para a etapa de definição da estratégia de busca, que consiste na definição das palavras-chave de pesquisa, que, intercaladas por operadores booleanos (AND, OR ou NOT) permitem a formação de String de busca automatizados de todos os termos nas bases de dados escolhidas.

Quadro 1 - Palavras-chaves para as Strings.

\begin{tabular}{|l|l|}
\hline \multicolumn{1}{|c|}{ Palavras-chave } & \multicolumn{1}{c|}{ key-words } \\
\hline Avaliação & assessment, evaluation \\
\hline Lógica de programação & logic programming \\
\hline Ensino médio & high school, secondary education, K-12 \\
\hline
\end{tabular}

A definição das palavras-chaves nos permitiu formar as seguintes Strings de busca: ((avaliação) AND ("lógica de programação" OR algoritmo) AND ("ensino médio")) para a busca nas bases de dados de Língua Portuguesa, e (("Assessment" OR "evaluation") AND ("logic programming") AND ("high school" OR "secondary education" OR "K-12")) para as bases de Língua Inglesa.

Para a realização das buscas, estabelecemos quais as bases de dados repositório dos estudos primários onde buscamos os estudos para a nossa pesquisa. Quanto às bases nacionais, elegemos as seguintes: Portal de Publicações da CEIE (RBIE; SBIE; WIE; WCBIE; JAIE; WDCAE; WAVEE), Revista Novas Tecnologias na Educação (RENOTE), Educação Profissional e Tecnológica em Revista e Revista Brasileira da Educação Profissional e Tecnológica. Em nível internacional, estabelecemos as seguintes bases de dados: ScienceDirect (Elsevier), Scopus Preview (Elsevier), IEEE Xplore, Wiley Online Library, Scielo (Web of Science) e EBSCO.

O trabalho de mapeamento busca estudos publicados nestas bases de dados entre o ano de 2008 e o ano de 2020. O critério para a escolha do ano de 2008 como período inicial do mapeamento se justifica pelo fato da promulgação da Lei $n^{\circ} 11.892$, de 29 de dezembro de 2008, que institui Rede Federal de Educação Profissional, Científica e Tecnológica e cria os Institutos Federais de Educação, Ciência e Tecnologia. A criação dos Institutos possibilitou a abertura de curso técnico de informática integrado ao ensino médio, o que motiva a pesquisa sobre metodologias de ensino e avaliação próprios para essa modalidade de curso integrado à educação básica. O ano de 2020 foi o período de qualificação do projeto de pesquisa.

A seguir, explicitamos os critérios de inclusão ou exclusão utilizados para classificar os estudos encontrados nas bases de dados escolhidas e que serão objeto de análise no presente estudo, conforme a tabela a seguir:

Quadro 2 - Critérios de inclusão e exclusão de material.

\begin{tabular}{|c|c|l|}
\hline Critério & Id & \multicolumn{1}{c|}{ Descrição } \\
\hline \multirow{4}{*}{ Inclusão } & I1 & Artigo completo publicado nas bases escolhidas. \\
\cline { 2 - 3 } & I2 & Estudos publicados entre o ano 2008 e o ano de 2020 \\
\cline { 2 - 3 } & I3 & $\begin{array}{l}\text { O estudo deve ter como temática principal a avaliação da } \\
\text { aprendizagem na área de lógica de programação. }\end{array}$ \\
\cline { 2 - 3 } & I4 & $\begin{array}{l}\text { Os trabalhos devem ter como foco a avaliação da aprendizagem } \\
\text { escolar na modalidade de ensino médio. }\end{array}$ \\
\hline & E1 & Artigos duplicados ou semelhantes \\
\hline
\end{tabular}




\begin{tabular}{|l|l|l|}
\hline \multirow{2}{*}{ Exclusão } & E2 & Artigos com acesso restrito \\
\cline { 2 - 3 } & E3 & Livros, dissertações ou teses \\
\cline { 2 - 3 } & E4 & Mapeamento/Revisão Sistemática \\
\hline
\end{tabular}

O próximo passo foi o acesso efetivo às bases de dados, que realizamos via acesso institucional remoto pelo Portal de Periódicos da Capes (Acesso CAFe), para garantir o alcance de maior quantidade de estudos. Acessamos a cada base de dados e pesquisamos a String, extraindo na sequência todos os estudos encontrados em cada base. Utilizamos os mecanismos de extração de metadados (título, autor, DOI, resumo, palavras-chave etc.) disponibilizados pelas próprias bases de dados. Esses metadados foram utilizados na primeira etapa (triagem) para escolha dos estudos que deveriam ser excluídos ou elegidos para análise do artigo completo. Na extração dos dados, foi aplicado o critério [I2] por meio da aplicação de filtro de temporalidade disponível nas próprias bases, o que nos permitiu selecionar somente os estudos publicados entre o ano de 2008 e o ano de 2020.

Realizada a extração dos dados, a próxima etapa consistiu na triagem dos estudos para incluí-los ou excluí-los, observando principalmente os critérios [I3] e [I4]. Esse processo de triagem foi realizado por meio da leitura do título e resumos dos trabalhos. Principalmente no resumo, deveria estar claro que aquele estudo tinha como objetivo principal a discussão sobre estratégias ou metodologias de avaliação da aprendizagem no ensino de lógica de programação para estudantes da educação básica.

Para realizar o registro mais sistemático das informações, utilizamos uma planilha do excel, onde foi possível registrar desde os quantitativos de estudos encontrados em cada base, assim como a classificação de cada estudo utilizando os metadados (autor, ano de publicação, revista, título, doi, link para o estudo e identificação da base de dados). Incluímos ainda três dados (resultados, inclusão/exclusão, critério de Inclusão ou exclusão) para preenchimento após a análise do título e do resumo de cada estudo.

\section{Resultados e Discussão}

As buscas foram realizadas no mês de janeiro de 2021. As buscas nas bases nacionais não nos trouxeram nenhum resultado. Nas bases internacionais, tivemos o retorno de 230 documentos no total e divididos por bases da seguinte forma: 40 na ScienceDirect, 6 na Scopus Preview, 157 na IEEE Xplore, 22 na Wiley Online Library, 2 no Scielo (Web of Science) e 3 no EBSCO.

O próximo passo foi a realização da triagem dos estudos, que consistiu na catalogação dos metadados de cada estudo numa tabela de excel e a leitura do título e resumo para classificá-los para a próxima etapa (etapa de confirmação), que consiste na leitura completa dos documentos. Nesta etapa, observamos os critérios de inclusão [I3] e [I4], bem como o critério de exclusão [E4]. Cabe ressaltar que os critérios [I1] e [I2] foram aplicados na própria etapa de busca e extração dos dados. Finalizada a etapa de triagem dos estudos, a totalidade dos documentos foi considerada inadequada para este estudo, pela ausência de uma discussão centrada nas estratégias ou modelos de avaliação da aprendizagem no ensino de lógica de programação para o Ensino Médio.

Mesmo trabalhos que traziam discussões sobre avaliação da aprendizagem, não a tinham como objeto central de discussão e análise, mas somente como atividade acessória utilizada para avaliação de determinada metodologia, prática de ensino ou mesmo uma intervenção tal como buscaremos exemplificar com os estudos abaixo.

Quadro 3 - Trabalhos com temática aproximada

\begin{tabular}{|l|ll|ll|}
\hline Autor & Título & Base & \\
\hline Ragonis (2009) & $\begin{array}{l}\text { Computing Pre-University: Secondary } \\
\text { Level Computing Curricula }\end{array}$ & $\begin{array}{l}\text { Wiley } \\
\text { Library }\end{array}$ & Online \\
\hline
\end{tabular}




\begin{tabular}{|l|l|l|}
\hline Haddadi et al. (2018) & $\begin{array}{l}\text { Peer assessment and groups formation in } \\
\text { massive open online courses }\end{array}$ & $\begin{array}{l}\text { Wiley Online } \\
\text { Library }\end{array}$ \\
\hline $\begin{array}{l}\text { De Melo et al. } \\
(2014)\end{array}$ & $\begin{array}{l}\text { Using arduino as pedagogical strategy in } \\
\text { learning logic programming }\end{array}$ & IEEE Xplore \\
\hline $\begin{array}{l}\text { Vosinakis, } \\
\text { Anastassakis e } \\
\text { Koutsabasis (2018) }\end{array}$ & $\begin{array}{l}\text { Teaching and learning logic programming } \\
\text { in virtual worlds using interactive } \\
\text { microworld representations }\end{array}$ & $\begin{array}{l}\text { Wiley } \\
\text { Library }\end{array}$ \\
\hline $\begin{array}{l}\text { Larraza-Mendiluze } \\
\text { et al. (2020) }\end{array}$ & $\begin{array}{l}\text { JolasMATIKA: An Experience for } \\
\text { Teaching and Learning Computing Topics } \\
\text { From University to Primary Education }\end{array}$ & IEEE Xplore \\
\hline $\begin{array}{l}\text { Cuevas-Martínez et } \\
\text { al (2019) }\end{array}$ & $\begin{array}{l}\text { Jump to the Next Level: A Four-Year } \\
\text { Gamification Experiment in Information } \\
\text { Technology Engineering }\end{array}$ & IEEE Xplore \\
\hline $\begin{array}{l}\text { Howard, Hyuk Park } \\
\text { e Remy (2012) }\end{array}$ & $\begin{array}{l}\text { Using Haptic and Auditory Interaction } \\
\text { Tools to Engage Students with Visual } \\
\text { Impairments in Robot Programming } \\
\text { Activities }\end{array}$ & IEEE Xplore \\
\hline
\end{tabular}

O trabalho de Ragonis (2009) trata de uma revisão sobre as diferentes abordagens para o ensino de computação em escolas de Ensino Médio no mundo. O trabalho de Melo et al. (2014) traz uma proposta de uso da plataforma arduino como estratégia pedagógica para o ensino de lógica de programação para estudantes do Ensino Médio e propõe uma avaliação da atividade com a finalidade de verificar se o uso da plataforma promoveu a aprendizagem. O texto de Vosinakis, Anastassakis e Koutsabasis (2018) trata da apresentação de uma plataforma de ensino e aprendizagem de Prolog que permite a verificação dos resultados de forma direta. O trabalho de Haddadi et al. (2018) propõe em método de avaliação por pares para MOOCs com o objetivo de melhorar a precisão das notas dos alunos. Larraza-Mendiluze et al. (2020) propõem uma metodologia de ensino e aprendizagem por meio de jogos que pode promover o conhecimento sobre ciência da computação para estudantes da educação básica. O artigo de Cuevas-Martínez et al. (2019) apresenta a experiência com gamificação para aumentar o engajamento e motivação dos estudantes. Já o trabalho de Howard et al. (2012) discute o uso de interfaces alternativas para envolver estudantes com deficiência visual nas atividades de programação baseadas em robótica.

Isso demonstra que, embora os trabalhos tratem de metodologias de ensino de programação e sua consequente avaliação, falta um questionamento centrado no aprofundamento teórico sobre as metodologias ou estratégias de avaliação da aprendizagem utilizadas no ensino de lógica de programação, ou seja, não houve discussão de diferentes instrumentos ou estratégias de avaliação e suas respectivas fundamentações teóricas ou qualquer discussão sobre a metodologia mais adequada a ser adotada. É necessário ter em consideração que a avaliação da aprendizagem é o que torna possível ao professor realizar o diagnóstico adequado das aprendizagens em sala de aula e, consequentemente, realizar o planejamento das intervenções pedagógicas necessárias para promover o avanço nas aprendizagens dos estudantes.

Os pesquisadores Raabe e Silva (2005) reconhecem essa dificuldade de avaliar no ensino de algoritmo e lógica de programação, e reforçam que a dificuldade de formular um diagnóstico das dificuldades de aprendizagem no período correto compromete a possibilidade de o docente traçar estratégias pedagógicas para promover a aprendizagem dos estudantes que possuem dificuldades, tendo como consequência a reprovação ou desistência. Eles concordam que "[...] o desenvolvimento de esforços para identificação 
e atendimento adequado as dificuldades de aprendizagem comuns aos alunos desta disciplina seja imprescindível." (RAABE; SILVA, 2005, p.2327)

\section{Conclusões}

O presente estudo teve como objetivo mapear na literatura científica as discussões sobre a avaliação da aprendizagem escolar no ensino de lógica de programação para estudantes do Ensino Médio. Esse tipo de estudo possui limitações que podem influenciar nos resultados da pesquisa. Uma dessas limitações é a própria circunscrição da pesquisa a determinadas bases de dados e a temporalidade, o que pode deixar trabalhos relevantes publicados em outras bases fora do estudo.

Nesta pesquisa, como nenhum estudo foi considerado adequado, não há como fazer uma discussão e análise a partir de propostas de avaliação da aprendizagem em lógica de programação formuladas por pesquisadores da área de avaliação ou ensino de programação, que é o objetivo principal. No entanto, o fato de não encontrar no mapeamento nenhum estudo adequado sobre o tema, fica claro que não há uma discussão dos pesquisadores de ensino de computação sobre as estratégias ou metodologias de avaliação da aprendizagem mais adequadas para a avaliação do ensino de programação no Ensino Médio. Isso pode indicar, por um lado, que ainda não há um questionamento científico sobre a efetividade das práticas de avaliação da aprendizagem correntes no ensino de programação por parte de professores e pesquisadores da área. Por outro lado, a ausência desse questionamento, pode ser um indício de que a avaliação é realizada pelos docentes de forma acrítica e repetitiva, sem base teórica e epistemológica definida, ou seja, é feita replicando estratégias ou métodos de avaliação utilizados pelos docentes dos cursos superiores, nos quais esses professores fizeram a graduação ou práticas aprendidas com colegas mais experientes, ou ainda montando os seus instrumentos de forma intuitiva, de acordo com uma consideração subjetiva sobre a forma mais adequada de avaliar a aprendizagem.

A área de ensino de programação é bastante complexa, tanto para o professor, que precisa se utilizar de uma linguagem adequada e metodologias diversas para conseguir fazer com que os estudantes compreendam os conceitos, quanto para os estudantes do Ensino Médio Integrado, que estão no período de desenvolvimento do pensamento formal e se apropriando dos conteúdos da educação básica, como matemática, lógica e física, que são considerados fundamentais para a aprendizagem de programação. Essa complexidade do ensino de programação demonstra a importância de pesquisar e discutir criticamente sobre a eficiência das estratégias e instrumentos de avaliação da aprendizagem no ensino de lógica de programação para a educação básica.

\section{Referências Bibliográficas}

BRITO, A.; MADEIRA, C. XP \& Skills: gamificando o processo de ensino de introdução a programação. Anais dos Workshops do Congresso Brasileiro de Informática na Educação, p. 1124-1133, 2015. ISSN 2316-8889. Disponível em: <https://brie.org/pub/index.php/wcbie/article/view/6235>. Acesso em: 23 ago. 2020.

CUEVAS-MARTÍNEZ, J. C.; YUSTE-DELGADO, A. J.; PEREZ-LORENZO, J. M.; TRIVIÑO-CABRERA, A. "Jump to the Next Level: A Four-Year Gamification Experiment in Information Technology Engineering," in IEEE Access, vol. 7, pp. 118125118134, Aug. 2019. Disponível em: https://ieeexplore.ieee.org/abstract/document/ 8786093. Acesso em: 13 jan. 2021. 
DE MELO, R. M.; DE OLIVEIRA FELICIANO, F. D.; BRITO, J. A.; DE MELO FILHO, I. J.; CARVALHO, R. S.; GOMES, A. S. "Using arduino as pedagogical strategy in learning logic programming," 2014 9th Iberian Conference on Information Systems and Technologies (CISTI), Barcelona, Spain, 2014, pp. 1-5. Disponível em: https://ieeexplore.ieee.org/document/6876894. Acesso em: 13 jan. 2021.

FORBELlONE, A. L. V.; EBERSPÄCHER, H. F. Lógica de programação: a construção de algoritmos e estruturas de dados. 3. ed. São Paulo: Pearson Prentice Hall, 2005.

HADDADI, L.; BOUARAB-DAHMANI, F.; GUIN, N.; BERKANE, T.; LAZIB, S. Peer assessment and groups formation in massive open online courses. Comput Appl Eng Educ.; v. 26: pp. 1873-1887. Sep. 2018. Disponível em: https://onlinelibrary.wiley.com/ doi/abs/10.1002/cae.22005. Acesso em: 13 jan. 2021.

HOWARD, A. M.; HYUK PARK, C; REMY, S. "Using Haptic and Auditory Interaction Tools to Engage Students with Visual Impairments in Robot Programming Activities," in IEEE Transactions on Learning Technologies, vol. 5, no. 1, pp. 87-95, First Quarter 2012. Disponível em: https://ieeexplore.ieee.org/document/6095498. Acesso em: 13 jan. 2021.

JESUS, A. D.; BRITO, G. S. Concepção de Ensino-Aprendizagem de Algoritmos e Programação de Computadores: A Prática Docente. I ENINED - Encontro Nacional de Informática e Educação, Cascavel, p. 132-141, 2009. ISSN 2175-5876. Disponível em: <http://www.inf.unioeste.br/enined/2009/anais/enined/A15.pdf>. Acesso em: 02 junho 2020.

JESUS, A. D.; BRITO, G. S. Concepção de ensino-aprendizagem de algoritmos e programação de computadores: a prática docente. Revista Varia Scientia, v. 9, n. 16, p. 149-159, Ago/Dez 2009. Disponível em: <http://e-revista.unioeste.br/index.php/ variascientia/article/view/2632>. Acesso em: 15 jun. 2020.

LARRAZA-MENDILUZE, E.; ARBELAITZ, O.; ARRUARTE, A.; LUKAS, J. F.; GARAY-VITORIA, N. "JolasMATIKA: An Experience for Teaching and Learning Computing Topics From University to Primary Education," in IEEE Transactions on Education, vol. 63, nº. 3, p. 136-143, Aug. 2020. Disponível em: https://ieeexplore.ieee. org/abstract/document/8915746. Acesso em: 13 jan. 2021.

LUCKESI, C. C. Avaliação da aprendizagem componente do ato pedagógico. 1. ed. São Paulo: Cortez, 2011.

MOREIRA, B. G. Desenvolvimento de uma ferramenta de avaliação por pares para disciplinas de algoritmos e programação. Anais dos Workshops do Congresso Brasileiro de Informática na Educação, p. 68-75, 2014. Disponível em: <https://www.br-ie.org/pub/index.php/wcbie/article/view/3173>. Acesso em: 13 jun. 2020.

OKOLI, Chitu. Guia para realizar uma revisão sistemática da literatura. Tradução de David Wesley Amado Duarte; Revisão técnica e introdução de João Mattar. EaD em Foco, 2019;9 (1):e748. DOI: https://doi.org/10.18264/eadf.v9i1.748

PERRENOUD, P. Avaliação: da excelência à regulação das aprendizagens - entre duas lógicas. Tradução de Pátricia Chittoni Ramos. Porto Alegre: Artes Médicas Sul, 1999.

PIMENTEL, E. P. et al. Avaliação Contínua da Aprendizagem, das Competências e Habilidades em Programação de Computadores. Anais do Workshop de Informática 
na Escola, p. 544-544, 2003. ISSN 2316-6541. Disponível em: <https://www.brie.org/pub/index.php/wie/article/view/819>. Acesso em: 5 jun. 2020.

RAABE, A. L. A.; SILVA, J. M. C. D. Um Ambiente para Atendimento as Dificuldades de Aprendizagem de Algoritmos. In: XXV Congresso da Sociedade Brasileira de Computação. São Leopoldo/RS, 2005.

RAGONIS, N. Computing Pre-University: Secondary Level Computing Curricula. In Wiley Encyclopedia of Computer Science and Engineering, B.W. Wah (Ed.). Mar. 2009. Disponível em: https://onlinelibrary.wiley.com/doi/abs/10.1002/9780470050118.ecse 974. Acesso em 13 jan. 2021.

RIBEIRO, A. L.; BITTENCOURT, R. A.; SANTANA, B. L. Análise da Motivação em um Estudo Integrado de Programação Baseado em PBL. Anais do XXVI Workshop sobre Educação em Computação, Porto Alegre, 26 jul. 2019. Disponível em: <https://sol.sbc.org.br/index.php/wei/article/view/3492>. Acesso em: 05 jun. 2020.

ROCHA, P. S. et al. Ensino e Aprendizagem de Programação: Análise da Aplicação de Proposta Metodológica Baseada no Sistema Personalizado de Ensino. RENOTE Revista Novas Tecnologias na Educação, v. 8, n. 3, p. sn, 2010. Disponível em: <https://seer.ufrgs.br/index.php/renote/article/view/18061>. Acesso em: 20 jul. 2020.

ROMÃO, J. E. Avaliação Dialógica: desafios e perspectivas. 9. ed. São Paulo: Cortez, 2011.

VOSINAKIS, S.; ANASTASSAKIS, G.; KOUTSABASIS, P. Teaching and learning logic programming in virtual worlds using interactive microworld representations. Br J Educ Technol, v. 49: p. 30-44. Dec. 2018. https://doi.org/10.1111/bjet.12531. Disponível em: https://bera-journals.onlinelibrary.wiley.com/doi/abs/10.1111/bjet.12531. Acesso em: 13 jan. 2021. 